INPLASY

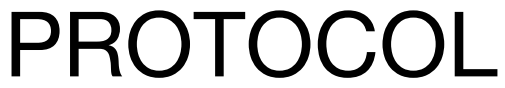

To cite: Zhang et al. A protocol for systematic review and meta-analysis of optimizing treatment for malaria. Inplasy protocol 202070115. doi: 10.37766/inplasy2020.7.0115

Received: 26 July 2020

Published: 26 July 2020

Corresponding author: Yong Dai

402975634@qq.com

Author Affiliation:

School of Basic Medicine,

Chengdu University of

Traditional Chinese Medicine.

Support: 3192018064.

Review Stage at time of this submission: The review has not yet started.

Conflicts of interest:

The authors declare no conflict of interest.

\section{A protocol for systematic review and meta-analysis of optimizing treatment for malaria}

Zhang, R; Dong, X²; Wang, J3; Guo, Y4; Dai, Y5.

Review question / Objective: P: People diagnosed with malaria. I: ACTs with either additional antimalarial agent or modified prescription in both medicine and course. C: Patients treating with ACTs or other recommended treatment. O: Zero parasitemia in peripheral blood smear by microscopy test and relief on malaria-related symptoms.

Condition being studied: Malaria is a parasitic disease through mosquito bites, which causes 400 thousand deaths globally every year. Even though WHO has recommended ACTs as standard treatment for uncomplicated malaria, an increasing number of treatment failures found in Southeast Asia worries people of global malaria control and eradication. Plasmodium falciparum is one of the human malaria species, which claims for the most morbidity and mortality, and is prone to develop resistance to current antimalarial agents.

INPLASY registration number: This protocol was registered with the International Platform of Registered Systematic Review and Meta-Analysis Protocols (INPLASY) on 26 July 2020 and was last updated on 26 July 2020 (registration number INPLASY202070115).

\section{INTRODUCTION}

Review question / Objective: P: People diagnosed with malaria. I: ACTs with either additional antimalarial agent or modified prescription in both medicine and course. C: Patients treating with ACTs or other recommended treatment. 0 : Zero parasitemia in peripheral blood smear by microscopy test and relief on malariarelated symptoms.

Condition being studied: Malaria is a parasitic disease through mosquito bites, 
which causes 400 thousand deaths globally every year. Even though WHO has recommended ACTs as standard treatment for uncomplicated malaria, an increasing number of treatment failures found in Southeast Asia worries people of global malaria control and eradication. Plasmodium falciparum is one of the human malaria species, which claims for the most morbidity and mortality, and is prone to develop resistance to current antimalarial agents.

\section{METHODS}

Participant or population: People diagnosed with malaria.

Intervention: ACTs with either additional antimalarial agent or modified prescription in both medicine and course.

\section{Comparator: ACTs.}

Study designs to be included: Randomized controlled trials or quasi-randomized controlled trials.

Eligibility criteria: Children over 5 years of age and non-pregnant adults who are diagnosed with uncomplicated malaria are eligible for our study.

Information sources: We will search electronic databases: the Cochrane Infectious Diseases Group Specialized Register, the Cochrane Central Register of Controlled Trials, MEDLINE, Embase, Web of Science up to July 1, 2020. We will also search the WHO International Clinical Trials Registry Platform and ClinicalTrials.gov, and contact with authors when necessary.

Main outcome(s): Patient's parasitemia in peripheral blood and parasite clearance time.

Quality assessment / Risk of bias analysis: The two investigators will independently evaluate the methodological quality of included literature with the Cochrane's risk of bias tool, Review Manager 5.3 (Revman V.5.3), according to CHSRI standard. The controversial studies will be submitted to the panel or consult with experts. Quality assessment will be performed and according to the Grading of Recommendations Assessment, Development, and Evaluation method (GRADE), covering the following aspects: random sequencing generation, allocation concealment, blinding of outcomes, completeness of outcome data, selective reporting, and other source of bias, such as conflicts of interest.

Strategy of data synthesis: According to the principle of PICO, we will draw a basic feature table of the included literature to make clear the basic information and differences in research methods, and to determine whether the data can be quantitatively synthesized.

Subgroup analysis: If necessary, a subgroup analysis of the included studies will be conducted with high heterogeneity, according to different intervention, administration and course.

Sensibility analysis: We will determine the stability of results by checking and by excluding the homogeneity among all included studies.

\section{Country(ies) involved: China.}

Keywords: malaria, Plasmodium falciparum, treatment, ACTs, review.

Contributions of each author:

Author 1 - Renyan Zhang.

Author 2 - Xing Dong.

Author 3 - Junyao Wang.

Author 4 - Ying Guo.

Author 5 - Yong Dai. 